\title{
Best Practices for the Con- servation and Preservation of Herbaria
}

\author{
Leora Bromberg
}

\section{Abstract:}

This paper offers an in-depth report on the best practices for the conservation and preservation of herbaria within library and museum collections. A herbarium (singular) is a collection of dried and pressed plant specimens, typically mounted onto paper and accompanied by a certain degree of recorded information. These organic specimens tend to be housed in museums or special collections libraries, where their handling can be carefully monitored and/or restricted. Each herbarium is typically one-of-a-kind and may serve as a vital primary source on human exploration, taxonomy, natural history and even amateur collection practices. A closer look at the best practices for their conservation and preservation spotlights the herbarium as a fragile, valuable and perhaps an unexpected or unusual form of "recorded information" that librarians, archivists and museum professionals may encounter or even have some responsibility over at some point in their careers.

\section{Keywords:}

Herbaria, natural specimens, rare books, conservation, preservation

DOI

10.33137/ijournal.v6i1.35263

(C) 2020 Bromberg, L. Best Practices for the Conservation and Preservation of Herbaria. This is an Open Access article distributed under CC-BY. (c) (i) 


\section{WHAT IS A HERBARIUM?}

A herbarium is a collection of dried and pressed plant specimens, typically mounted onto paper and accompanied by a certain degree of recorded information. These collections have traditionally appeared in the form of bound volumes, or as collections of individual sheets, which has become the standard form for modern herbaria (Massey, 1974). Plant specimen collection practices have ranged over time and place from domestic albums compiled by private or amateur collectors to rigorous scientific collections, known as exsiccatae, often collected by corporate bodies such as universities and government agencies, which may include millions of specimens (Cooper, 2018, p. 268; Massey, 1974; Zytaruk, 2015). In either case, specimens are traditionally mounted in such a way that displays as many distinguishing features of a plant as possible without misconfiguring or damaging its natural structure (Zytaruk, 2019, p. 13; Collins, 2014). Whether bound or unbound, the herbarium has come to be considered a vital reference system in itself in supporting ongoing plant identification, developments in taxonomy, and natural history education and research (Collins, 2014).

The herbarium in any form can justly be considered part of the realm of "recorded information," as these collections serve as primary sources on human exploration, taxonomy and natural history. Whether featured in a scientific or amateur collection, each specimen is typically accompanied by information, such as the species, taxon, location of discovery, date, and/or personal notes (Massey, 1974). The recorded data accompanying any given specimen is as important as the specimen itself, adding significant and often irreplaceable item-level description and thereby scientific, historical, or social value to the collections (Collins, 2014). Herbaria also serve important research purposes. For instance, these collections facilitate the ongoing systematic study of plants even beyond the blooming season. The samples also allow for tangibility and accuracy in study, serving as a useful alternative to botanical illustrations (Cooper, 2018, p. 268). Furthermore, the preservation of plants as actualized through herbaria may become especially valuable in face of global crises such as climate change, as habitats suffer major transformations and some plant species face the threat of extinction. 
Herbaria bound in volumes, especially those of an early date or historical significance, may typically be found in museums or special collection libraries, where their handling can be carefully monitored or restricted. The University of Toronto's Thomas Fisher Rare Book Library holds a rather diverse collection of herbaria, from books with plants casually laid in to amateur albums, seaweed albums and scientific bound volumes of plant specimens. Loose-leaf herbaria, on the other hand, tend to be collected by museums or government agencies. Often, these collections are not just historical, but may continue to grow as new samples are added. Some notable large-scale and long-developed local collections include the National Herbarium of Canada at the Canadian Museum of Nature, and the ROM's Green Plant Herbarium. The National Herbarium of Canada includes a diverse collection of vascular plants, bryophytes, lichens and algae that amount to well over one million specimens, and the herbarium at the ROM is likely "the largest and most representative collection of the Ontario flora" (Canadian Museum of Nature, n.d.; University of Toronto, n.d., n.p.).

\section{A BRIEF HISTORY OF HERBARIA}

Although there is some evidence that members of ancient Greek society, known as the botanologoi, devoted themselves to gathering plants, herbaria in the form of bound or unbound paper volumes did not emerge until towards the end of the sixteenth century. At this time, the term hortus siccus, or "dry garden," designated what we would now refer to as a herbarium (Torrey Botanical Society, 1885, p. 129-130).

Curiously, the history of herbaria greatly relied on developments in paper production over time. The relatively later emergence of herbaria makes sense considering that ancient writing surfaces, such as parchment and papyrus, were typically costly to produce and formatted as scrolls as opposed to flat or heavy codices. These early writing surfaces would therefore not have served practically for plant preservation. The longevity of pressed plants relied on identifying a convenient and affordable mounting material. Indeed, herbaria became more common as papermaking practices shifted towards the use of linen and hempen rags in the fourteenth century and iJournal, Vol 6, No. 1, 
as machines for printing and papermaking emerged in the mid-fifteenth century (Torrey Botanical Society, 1885, p. 130-131). Overall, long-term plant preservation in many ways came to depend on developments in papermaking techniques and the format of the codex.

Printed books with mounted specimens were especially popular during the Victorian period. These books with "natural illustrations" appear for sale in nineteenth-century bookseller catalogues (Zytaruk, 2015, p. 13). This market reflects a particularly Victorian desire to collect natural objects. In fact, it is thought that as a young girl, Queen Victoria participated in this plant-collecting culture by compiling a seaweed album of her own (Zytaruk, 2019, p. 8). There were even instruction manuals on collecting and preserving botanical specimens, which targeted both scientific and amateur readerships at the time. One such manual was William Withering's A

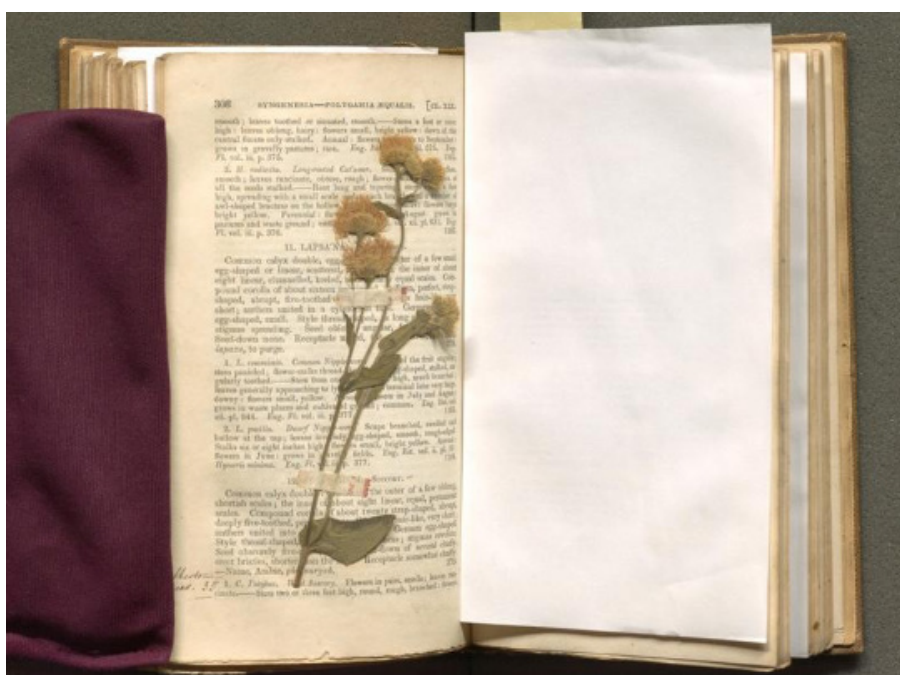

Figure 1: William Withering's A Systematic Arrangement of British Plants (1835, 3rd ed) held at the Fisher Library. This copy features 15 pressed specimens laid-in and marginal notes. Acid-free paper is also laid-in to indicate the location of specimen throughout the volume and to protect adjacent pages. Courtesy of Thomas Fisher Rare Book Libary, University of Toronto.
Systematic Arrangement of British Plants

(1776) which was reprinted in several different editions and formats (Figure 1). Withering's manual instructs readers on how to compile and maintain a herbarium of their own and also provides a dictionary of botanical terms (Zytaruk, 2019, p. 11). According to Zytaruk (2019), Withering's manual captures the "paper-heavy nature of this kind of collection" and the technical expertise required to maintain it, suggesting that even nineteenth-century botanists were concerned about the longevity and preservation of their samples (p. 12). Withering's manual also helped to popularize the system of plant identifi-

cation developed by Carl Linnaeus (Zytaruk, 2019, p. 11). Linnaeus was a leading voice in advocating for a shift from bound-volume to single-sheet herbaria. As he argued, single-sheet storage 
proved advantageous for long-term preservation given that in this format it is easier to control any movement inflicted upon the specimens as well as maintain and update their taxonomic order (Collins, 2014).

\section{MAIN THREATS TO HERBARIA LONGEVITY}

Herbaria generally face many of the same risks to their longevity as any book and paper material. Like any paper materials, herbaria may be damaged or destroyed by fire, water, fluctuations in temperature and relative humidity, pollutants, light, theft, pests and poor handling and storage. Typically, the most common forms of damage to plant specimens are breakage and loss as a result of poor handling and storage practices (Graham, 2018). Dried plants and even their mounting adhesive or paper may become extremely brittle and fragile and any breaks or losses may risk significantly decreasing a sample's aesthetic and research value. Their small size and fragility may also render these collections more vulnerable to theft and vandalism (Graham, 2018). Given their added organic matter, herbaria are highly susceptible to pests, particularly insects including the larvae of cigarette and carpet beetles, moths, booklice and silverfish (Massey, 1974).

Preservation techniques for herbaria may vary depending on the type of specimens featured in the collection. For instance, bryophytes, which include mosses, liverworts and hornworts, and lichens, must be treated separately from vascular plants such as seed plants and ferns (British Columbia Ministry of Forests, 1996, p. 1). Generally speaking, specimens that are stored and handled appropriately can be preserved for a hundred years or more (British Columbia Ministry of Forests, 1996, p. 19).

The distinct features and conditions of the specimens within the collection will also dictate their handling and accessibility. For instance, herbaria may include endangered species, which require extra attention and sensitive handling given that they are of scientific value and are often irreplaceable (Massey, 1974). Furthermore, when dealing with dried plant collections, it is also critical to prioritize and be mindful of health and safety. Some specimens may include sharp stems 
or thorns that may cause harm during handling. In some cases, herbaria may even include specimens of poisonous or allergenic plants. As a result of now outdated conservation practices, some samples may have also been treated with toxic insecticides such as arsenic or mercury compounds

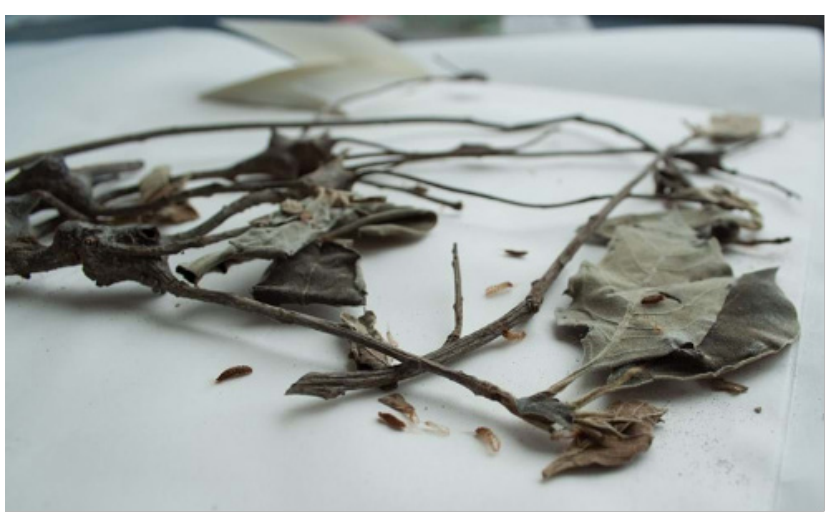

Figure 2: An example of insect damage -larvae skins on specimen (Royal Botanic Garden of Edinburgh, n.d.).
(Collins, 2014; Graham, 2018). Since these contaminants may cause severe irritation, it is imperative that specimens with such treatments are marked as hazardous and handled with appropriate protective equipment.

\section{BEST PRACTICES FOR HER- BARIUM PRESERVATION}

\section{Pest prevention and treatment}

Unless specimens have been previously treated with pesticides, these collections should be considered highly vulnerable to insects (Figure 2) (Graham, 2018).

A number of methods have traditionally been used to prevent or get rid of insect pests in herbaria, including poisoning, fumigation and heating (Massey, 1974). However, each of these methods have been deemed to be ineffective for herbarium preservation and raise many potential health hazards (British Columbia Ministry of Forests, 1996, pp. 20-21). Pesticides may significantly weaken and embrittle paper and blacken labels, and fumigation often fails to eliminate insect eggs (Massey, 1974). Any specimens that have been previously treated with poisonous compounds that may put researchers at risk must be clearly labelled, as a health and safety measure. As a precaution to avoid inflicting any unnecessary or irreversible damage on specimens, conservators should not attempt to eliminate historic pesticide treatments or apply any new chemicals (Collins, 2014). Lastly, heating has also proven to be harmful to the dried plant specimens themselves, as this process risks causing degradation of their DNA (Collins, 2014).

The most common and effective method of pest control is freezing. For loose-leaf herbaria, specimens should be frozen on a rotating schedule after being dried, acquired or returned from iJournal, Vol 6, No. 1, 
loan (British Columbia Ministry of Forests, 1996, p. 21; Collins, 2014). The specimens must be properly packaged when frozen and allowed about a day before and after freezing to acclimatize before being reintroduced to the collection. Otherwise, freezing will introduce condensation and moisture, which will weaken the dried plants and create an environment conducive to pests and mould. To prevent freezer burn, specimens should be sealed tightly in plastic bags, and should be further supported with cardboard between sheets. The bags should be carefully stacked in a standard chest freezer at $-20^{\circ} \mathrm{C}$ for $48-72$ hours (British Columbia Ministry of Forests, 1996, p. 21; Collins, 2014).

\section{Temperature, relative humidity and light exposure}

Herbaria should be stored in a secure and climate-controlled building, with a stable temperature of about $20^{\circ} \mathrm{C}$ and a relative humidity of $50 \%$. Fluctuations in temperature and relative humidity can damage fragile plant specimens (Royal Botanic Garden of Edinburgh, n.d.). A relative humidity of $65 \%$ or higher can be conducive to mould and pests, and in dry conditions, with a relative humidity under $30 \%$, specimens can become embrittled and more susceptible to damage during handling. The storage facility's central HVAC (heating, ventilation and air conditioning) system should be set up with appropriate filters to reduce and control the volume of dust and other pollutants in the environment (Graham, 2018).

Dried organic specimens are typically also at a medium-to-high risk of damage from exposure to visible and ultraviolet (UV) light. Exposure to light may result in fading, discolouration, and embrittlement in plant specimens. This damage may be observed on samples that have been on long-term display (Graham, 2018). To prevent light-related damage, natural specimens should not be featured in permanent exhibitions, but, if necessary, may be displayed temporarily or on a rotation. Storage units for these collections should therefore be located out of reach of natural daylight and UV light should be eliminated from all light sources (Graham, 2018). All of these factors including temperature, relative humidity, air quality and lighting conditions within the herbaria storage, display and study environment should be regularly and carefully monitored and recorded iJournal, Vol 6, No. 1, 
by one or more staff members (Graham, 2018).

\section{Storage}

Bound volume herbaria may be shelved among other books, within enclosures, or with ties as necessary, depending on the condition of their binding and paper. It may be necessary to shelve bound volumes horizontally, depending on the condition of the specimens and their adhesive or lack thereof within. Proper storage

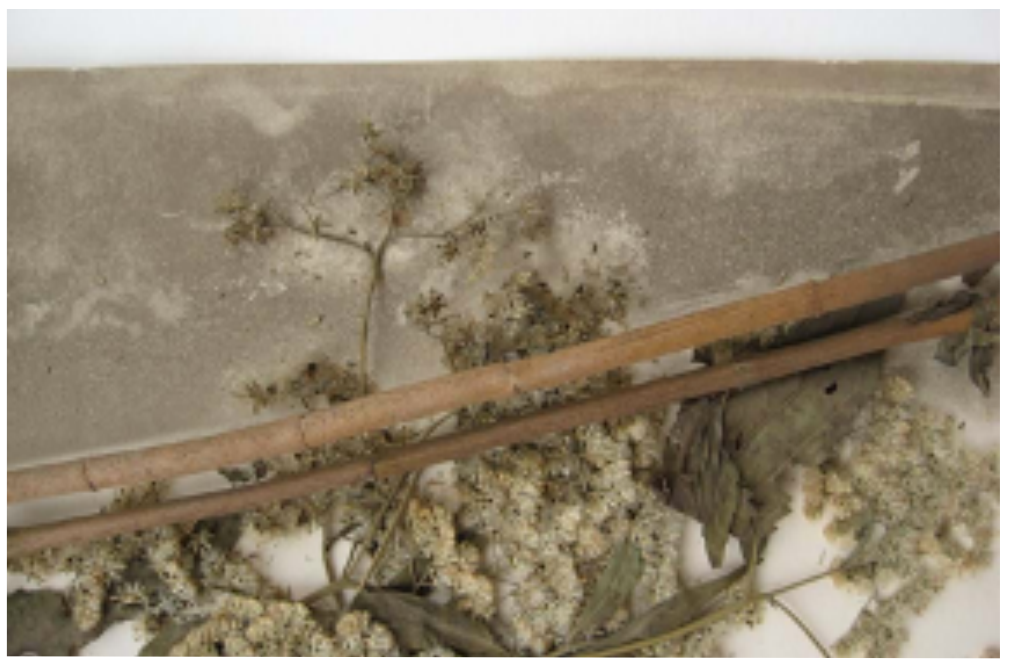

Figure 3: An example of poor storage -dusty specimen (Royal Botanic Garden of Edinburgh, n.d.). for loose-leaf herbaria involves thoughtful organization, handling and maintenance. For loose-leaf collections, specimens should be mounted on thick and supportive paper, and stored individually in large acid-free folders called "genus covers," or in boxes made of plastic board or acid-free matboard (Graham, 2018; Massey, 1974). File storage is crucial in protecting specimens from accumulating dust

(Figure 3). Botanical specimens need to be protected from dust, as their fragility makes them very challenging to clean and dust also attracts pests (Graham, 2018). If dust or dirt has already accumulated on or around the surface of a specimen, the appropriate cleaning method involves using a chemical sponge and a soft brush, though this process typically requires some expertise and training in order to avoid causing any additional damage (University of Aberdeen, 2017).

Filed specimens should be stored on powder-coated steel shelves within metal cabinets. The cabinets should ideally include rubber door seals that protect the items from exposure to light, dust and insects (Royal Botanic Garden of Edinburgh, n.d.). In order to further minimize any risk of damage, the cabinets should be robust and open and close smoothly. Metal is preferable to wood 
for storage and display units as wood products may increase the risk of pest infestation (British Columbia Ministry of Forests, 1996, p. 19; Graham, 2018). Any vulnerable and brittle items should be stored in appropriate enclosures, labelled as fragile, and have restrictions on their access and display, and those that are especially light-sensitive should be kept in dark storage in opaque containers (Graham, 2018).

\section{Handling}

Access and handling are essential aspects of an active research herbarium, but with usage comes risks of damage. It is essential to have policies, procedures, and training in place for both staff and visitors in order to ensure that herbaria are handled with care and that all parties are safe (Collins, 2014). This may be accomplished through regular staff training, visitor contracts, or posters and pamphlets that reinforce the handling policies. Many institutions will also opt to have researchers supervised when studying the collections (Royal Botanic Garden of Edinburgh, n.d.).

Bound volume plant specimens are especially sensitive to damage caused by careless handling, as these books tend to be older and prone to buckling pages and tight or deteriorating bindings (Zytaruk, 2015, p. 13). In the form of a book, the specimens are at risk of shifting or breaking, as they cannot be studied without their pages being turned and potentially warped. The accessibility and availability of these collections is often impacted by their bindings, which may over time become significantly weakened and require conservative repair (University of Aberdeen, 2017).

Both bound volumes and loose-leaf herbaria should be carried horizontally with two hands and transported longer distances with the help of a book truck. When handling loose-leaf specimens, the individual sheets should not be flipped like the pages of a book, but rather lifted right side up using two hands and placed back inside folders. Handling policies, training sessions, and supervision should effectively communicate to staff and visitors the importance of reporting any specimens that show signs or potential risk of damage (British Columbia Ministry of Forests, 1996, p. 20). It is also worth noting that heavy objects or books should never be placed on top of specimen folders or even bound volume specimens (Collins, 2014). Generally, specimens should iJournal, Vol 6, No. 1, 
be handled as infrequently as possible, especially those that are particularly fragile and brittle. Appropriate safety equipment, such as shallow trays, gloves, and masks should also be made available to protect users from any toxic residues that may be present on specimens marked as hazardous (Collins, 2014).

\section{Dissociation}

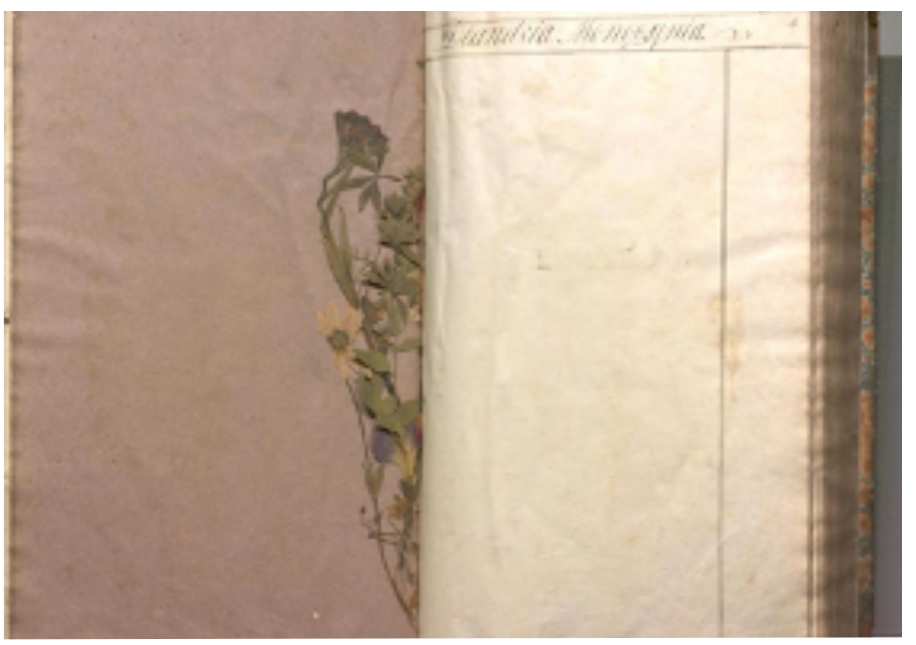

Figure 4: Alexander Thomson's Hortus Conservatio, or Garden Preservation of Plants Indigenous and Exotic with their Generic and Specific Characters, Places of Growth, Paration, Times of Flowering [manuscript] (1823) held at the Fisher Library. This page of Thomson's herbarium is presumably an example of the consequences of poor handling and age. The plant specimens on this page remain loose and have shifted, causing some pieces to suffer breakages. Courtesy of Thomas Fisher Rare Book Libary, University of Toronto.
Even in stable or ideal environmental conditions, dried plant specimens and their mounting paper or adhesive may become particularly weak and fragile over time (Figure 4). Any small or loose samples such as seeds or fragments should remain with the original

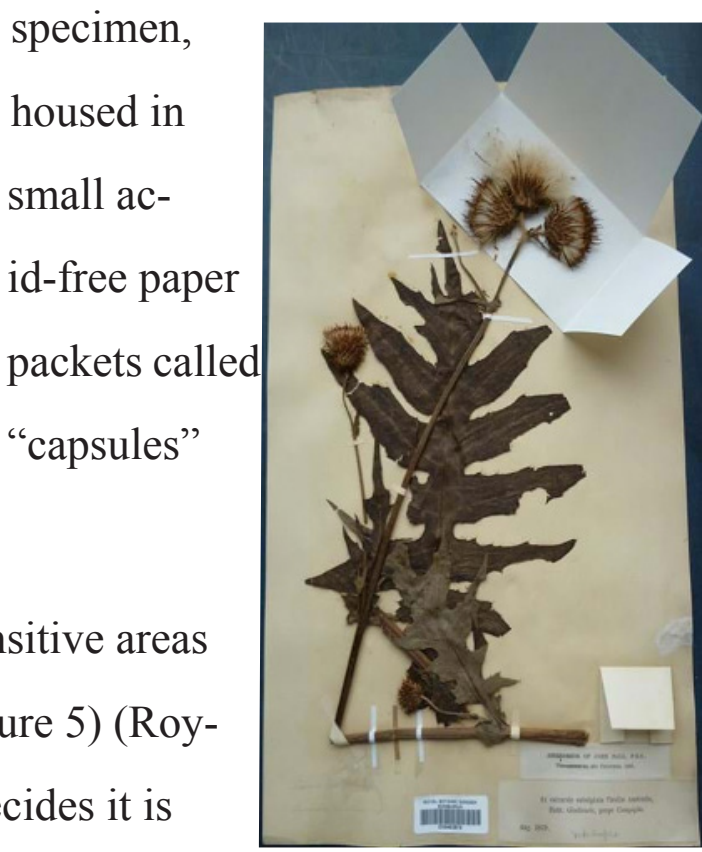

Figure 5: Capsule added to protect fragile flower (Royal Botanic Garden of Edinburgh, n.d.) rioration. Appropriate methods of adhesion include strapping with linen tape, gluing with gelatine or methyl cellulose, or stitching with thread. Any means of adhesion 
should be applied to the specimen in a gentle manner and should always be reversible (Collins, 2014).

\section{Data and digital preservation}

As mentioned earlier, herbaria draw much of their value from the information and data that accompanies their samples. Therefore, their preservation is incomplete without proper efforts to preserve labels, annotations, and field or personal notes associated with the specimens. Most importantly, labels or accompanying information should always remain with the appropriate specimens. In the case of replacing, remounting, or creating new labels for specimens, the label, like the mounting paper itself, should be printed on acid-free paper. Neither the plant nor its accompanying labels should be remounted if the object as a whole is deemed to be of historical significance, as this process may not only risk damaging the specimen, but the original arrangement, label(s), and even the mounting paper itself may also hold or add some historical significance from a heritage standpoint (Collins, 2014). Data preservation efforts for herbaria should also extend into the digital realm. The data retrieved from specimens should be made accessible through an electronic collections management database that undergoes sufficient and regular backups (Collins, 2014). A herbarium should ideally also be accompanied by a printed guide or finding aid, which serves to help staff and users navigate classifications, any colour-coding applied to folders and other storage details and collection highlights (Massey, 1974).

In addition to databasing specimens, some institutions have also made efforts towards preserving herbaria digitally. For example, the Thomas Fisher Rare Book Library has several publicly accessible Flickr albums dedicated to highlighting various mounted specimens that can be found within their collection (Thomas Fisher Rare Book Library, 2016). Digital scans can be an effective and advantageous method to increase access while minimizing handling and also capturing the original condition of the specimens. This method of preservation may prove to be especially important when dealing with the preservation of specimens belonging to rare, threatened, or extinct species. 


\section{CONCLUSION}

Although the sale of herbaria in bound volumes and the desire to collect and dry plants has more or less fallen out of popular practice, these collections remain a major and curious part of book history and thereby library and museum collections. The modern herbaria in the form of loose-leaf specimen sheets remains a central and ongoing aspect of botanical and natural history research. Though there have been some improvements, especially from the perspective of prioritizing health and safety, the general practices for the conservation and preservation of both bound and unbound herbaria have not changed all that much since Withering's time, when specimen collectors and botanists had the forethought to express concern for the long-term preservation of their samples as well as their accompanying information (Zytaruk, 2019, p. 12). Since plant specimens are particularly vulnerable to damage from poor storage and handling, and considering the fact that some specimens may even be irreplaceable, the best way to ensure their preservation is through proactive and preventative practices. Preventative preservation for herbaria includes periodic monitoring of the collection's storage and environmental conditions, as well as the physical condition of specimens, repair or re-casing as necessary, and the establishment of clear handling and access policies. While this report has aimed to outline the best practices to-date for the preservation and conservation of herbaria within library and museum institutions, each collection may raise unique challenges or exceptions depending on the types of plants included, as well as the format and overall condition of the collection. These best practices will also continue to develop over time as new findings, techniques, and technologies for the preservation and conservation of herbaria emerge. With this in mind, in order to make the best and most precautious decisions for the conservation of herbaria, it may be useful to consult several different experts as well as keep up with emerging best practices within the field (University of Aberdeen, 2017). Every specimen and every herbarium is unique; therefore, their preservation may require tailored approaches. 


\section{References}

British Columbia Ministry of Forests. (1996). Techniques and procedures for collecting, preserving, processing, and storing botanical specimens (pp. i-vi, 1-39). https:/www.for.gov.bc.ca/hfd/ pubs/docs/wp/wp18.pdf

Canadian Museum of Nature. (n.d.). "Botany Collections: The National Herbarium of Canada." https://nature.ca/en/research-collections/collections/plants-algae

Collins, C. (2014). "Standards in the care of botanical materials." The Conservation Centre, National History Museum, London. http://conservation.myspecies.info/node/35\#

Cooper, A. (2018). Placing plants on paper: Lists, herbaria, and tables as experiments with territorial inventory at the mid-seventeenth-century Gotha court. History of Science, 56(3), 257-277.

Graham, F. (2018). "Caring for natural history collections-Preventive conservation guidelines for collections." Canadian Conservation Institute.

https://www.canada.ca/en/conservation-institute/services/preventive-conservation/guidelines-collections/natural-history.html

Massey, J. R. (1974). Chapter 31: The herbarium. In Vascular Plant Systematics by A. E. Radford, W. C. Dickison, J. R. Massey and C. R. Bell. https://www.ibiblio.org/unc-biology/herbarium/ courses/chpt31.html

Royal Botanic Garden of Edinburgh. (n.d.). "Care and conservation of herbarium specimens." https://www.rbge.org.uk/science-and-conservation/herbarium/specimen-preparation-care/ care-and-conservation-of-herbarium-specimens/

Thomas Fisher Rare Book Library. (2016). Mounted specimens in the nineteenth-century book. Flickr. https://www.flickr.com/photos/thomasfisherlibrary/albums/72157668758164573

Thomson, A. (1823). Hortus conservatio, or Garden preservation of plants indigenous and exotic with their generic and specific characters, places of growth, paration, times of flowering [manuscript]. England. [Thomas Fisher Rare Book Library call no.: MSS 09708].

Torrey Botanical Society. (1885). The Origin of Herbaria. Bulletin of the Torrey Botanical Club, 12(12), 129-131.

University of Aberdeen. (2017, November 13). "Leaves of leaves: Conservation and housing of a book of Sub-Himalayan fern specimens."

https://specialcollectionslearning.wordpress.com/2017/11/13/leaves-of-leaves-conservation-andhousing-of-a-book-of-sub-himalayan-fern-specimens/

University of Toronto, Dept. of Ecology and Evolutionary Biology. (n.d.). "ROM: Green Plant Herbarium." http://www.eeb.utoronto.ca/research/facilities/ph-rom.htm

Withering, W. (1835). A systematic arrangement of British plants. London: Printed for Scott, Webster, and Geary... [Thomas Fisher Rare Book Library call no.: sci 06744].

Zytaruk, M. (2015). Mary Howard's ocean flowers (the Victorian taste for natural illustrations). The Halcyon: The Newsletter of the Friends of the Thomas Fisher Rare Book Library, (55), 1315.

Zytaruk, M. (2019). Nature on the Page: The Print and Manuscript Culture of Victorian Natural History. Thomas Fisher Rare Book Library exhibition 28 January to 26 April, 2019. Toronto: The Thomas Fisher Rare Book Library, University of Toronto. 